\title{
Lidil
}

Revue de linguistique et de didactique des langues

\section{Étude lexicosémantique du nom difficulté(s) dans les comptes rendus de bilan orthophonique : apports structuraux et conceptuels}

Lexical and Semantic Description of the Noun Difficulty(ies) in Speech and Language Therapy Reports: Structural and Conceptual Findings

Frédérique Brin-Henry et Marie Laurence Knittel

\section{OpenEdition}

Journals

Édition électronique

URL : http://journals.openedition.org/lidil/3915

DOI : 10.4000/lidil.3915

ISSN : $1960-6052$

Éditeur

UGA Éditions/Université Grenoble Alpes

Édition imprimée

Date de publication : 30 mai 2016

Pagination : 19-41

ISBN : 978-2-84310-326-1

ISSN : $1146-6480$

\section{Référence électronique}

Frédérique Brin-Henry et Marie Laurence Knittel, «Étude lexicosémantique du nom difficulté(s) dans les comptes rendus de bilan orthophonique : apports structuraux et conceptuels », Lidil [En ligne], 53 2016, mis en ligne le 01 janvier 2017, consulté le 29 octobre 2020. URL : http:// journals.openedition.org/lidil/3915; DOI : https://doi.org/10.4000/lidil.3915 


\title{
Étude lexicosémantique du nom difficulté(s) dans les comptes rendus de bilan orthophonique : apports structuraux et conceptuels
}

\author{
Frédérique Brin-Henry* et Marie Laurence Knittel**
}

\begin{abstract}
RÉSUMÉ
Une étude du nom difficulté(s) et son environnement dans un corpus de comptes rendus de bilans orthophoniques français a été entreprise afin d'explorer conjointement les contraintes syntaxiques et les variations sémantiques du contexte droit des occurrences. Cet intérêt se justifie par la présence particulièrement fréquente de ce nom dans le corpus. Cet article rend compte des étapes méthodologiques et des résultats selon trois axes : syntaxique, sémantique et disciplinaire. Ainsi, nous montrons que ce sont les adjectifs taxinomiques («difficultés articulatoires ») qui sont les plus fréquemment utilisés pour spécifier les difficultés. Par ailleurs les variantes («difficultés scolaires», «difficultés persistantes », «plus globales») donnent des informations à la fois sur le type de difficultés et la façon dont elles s'expriment chez le patient. Enfin, la variation dans les routines discursives («difficultés à accéder »vs «difficultés d'accès ») permet de différencier certaines modalités d'expression de la pathologie. Cette étude alimente nos travaux d'exploration des représentations des pathologies du langage chez les orthophonistes.
\end{abstract}

\section{ABSTRACT}

This study deals with the noun "difficulty(ies)" and its environment in a corpus made out of French speech and language therapy (SLT) reports. It explored the syntactic constraints and the semantic variations of the right context of each occurrence. This seemed relevant since this noun appeared very frequently in the corpus. This article details each methodological step we chose, along with the results according to

* Centre hospitalier de Bar-le-Duc, UMR 7118 ATILF, Université de Lorraine.

** UMR 7118 ATILF, Université de Lorraine. 
the syntactic, semantic and disciplinary axes. For instance, mostly taxonomic adjectives ("articulatory difficulties") are used to describe the difficulties. Variations ( "school difficulties", "persistent difficulties", "global difficulties") designate how the difficulties are and how they appear in patients. Finally, variations in routine formulae ("access difficulties" vs "difficulties to access") help speech and language therapists to differentiate between types of pathologies. This contributes to a better understanding of SLT representation of language pathology.

\section{Introduction}

L'objectif de ce travail est d'étudier le nom difficulté(s) et son environnement dans un corpus de comptes rendus de bilans orthophoniques (dorénavant CRBO) anonymisés constitué en 2007 à des fins terminologiques (Brin-Henry, 2011b). L'inconstance diachronique et synchronique des termes utilisés par les orthophonistes (voir section 2.1) justifie un examen approfondi des pratiques onomasiologiques qui permettent entre autres l'étiquetage des troubles du langage (Walsh, 2007). De plus, le traitement des données extraites permet l'examen de la langue de spécialité. Le nom difficulté( $(s)$ apparait fréquemment dans ce corpus, soit globalement dans le texte du compte rendu, soit plus précisément dans les termes diagnostiques ${ }^{1}$ utilisés, comme en témoignent les exemples (1) :

(1)

a. Madame a rencontré de nombreuses difficultés vocales tout au long de ce bilan.

b. Écriture très ralentie avec des difficultés à effectuer les boucles.

c. Le test des cloches met en évidence des difficultés de stratégie.

d. Les difficultés en calcul persistent.

À partir d'un inventaire des occurrences rencontrées et de leurs contextes, nous nous attachons à déterminer les moyens d'expression propres à la langue de cette spécialité, à en caractériser les structures sur le plan formel, et à repérer leurs éventuels emplois spécifiques, au sein

1. Un terme diagnostique est une unité terminologique utilisée pour désigner les collocations et les locutions (aphasie, trouble d'articulation), ainsi que les syntagmes (troubles d'analyse phonologique et de mémoire auditive) ayant servi à l'orthophoniste pour étiqueter les troubles ou difficultés mis en évidence au cours du bilan orthophonique (Brin-Henry, 2011b). 
de possibles routines discursives (Née, Sitri \& Veniard, 2014). Nous situant plus spécifiquement entre les domaines de l'analyse du discours et de la sémantique de corpus (Condamines, 2007) à des fins terminologiques (L'Homme, 2008), nous nous interrogeons également, à la manière de Veniard (2008), sur ce que l'emploi du terme difficulté(s) révèle des intentions et des représentations de l'orthophoniste. Selon nous, l'usage conventionnel du CRBO en fait un genre discursif spécifique à la profession, disposant de ses propres routines et patrons. Au-delà de la variation individuelle, nous pensons que les types syntaxiques et sémantiques mettent en lumière des conceptions ou des représentations spécifiques que ces professionnels ont des pathologies du langage.

Notre article s'organise comme suit. Après un rappel des propriétés linguistiques et terminologiques de difficultés (section 2), nous exposons nos choix méthodologiques dans la section 3. L'étiquetage syntaxique et sémantique que nous avons opéré est présenté en section 4. Le croisement de ces deux paramètres nous offre une grille de présentation de nos résultats (section 5.1), qui sont ensuite (section 5.2) questionnés à la lumière de la discipline «orthophonie», notamment en regard de la valeur des variations observées. La section 6 conclut notre travail en présentant les perspectives ouvertes par nos résultats.

\section{Pourquoi le nom difficultés?}

\subsection{Le nom difficultés : propriétés linguistiques}

Difficultés est un nom désadjectival construit sur l'adjectif difficile, qui présente trois structures :

(2)

a. Il est difficile [de \{travailler/résoudre ce problème\}].

b. \{Travailler/résoudre ce problème\} est difficile.

c. Ce problème est difficile à résoudre.

La structure (2a) est la variante impersonnelle de la structure (2b), fréquemment observée tant pour des verbes, dit inaccusatifs (cf. une catastrophe est arrivée / il est arrivé une catastrophe) que pour des adjectifs (cf. fuir était stupide / il était stupide de fuir), appelés adjectifs ergatifs; la construction (2c), dans laquelle l'objet du verbe à l'infinitif (i.e. ce problème) initialement complément de l'adjectif apparait comme sujet, est beaucoup moins fréquente, et ne concerne qu'une petite 
classe d'adjectifs sémantiquement proches (simple, aisé, compliqué, dur, etc.; voir à ce propos Soare \& Dobrovie-Sorin, 2002; Soare \& Giurgea, 2007).

Quant au nom désadjectival difficulté, il se présente comme un nom abstrait, paraphrasable par ce qui est difficile; les exemples (2) recevront donc, au moyen du nom difficulté(s), les paraphrases en (3); on notera que la préposition de, employée pour introduire la subordonnée complément de l'adjectif (cf. (2a)) alterne avec à (voir section 5) lorsque la subordonnée dépend du nom (3a).

(3)

a. La difficulté \{à/ ??de\}\{travailler/résoudre ce problème\} [comp. (2a)]

b. \{Travailler/résoudre ce problème\} est une difficulté [comp. (2b)].

Comme la plupart des noms désadjectivaux, difficulté(s) désigne la/ les entité(s) caractérisée(s) par la propriété décrite par l'adjectif de base. Ainsi, une difficulté est foncièrement ce qui est difficile, ou est perçu comme tel, d'où l'emploi fréquent comme objet de verbes supports de type éprouver, connaitre, montrer, manifester, etc., qui introduisent typiquement les noms de sentiments et d'attitude (Anscombre, 1995). Sur le plan sémantique, cette entité s'interprète également comme la cause de la ou des difficultés; c'est ce que montre l'équivalence sémantique des exemples en (4):

(4)

a. \{Travailler/résoudre ce problème\} est \{difficile/une difficulté\}.

$=\mathrm{b} .\{$ Travailler $/$ résoudre ce problème $\}$ cause $\{$ une/des $\}$ difficulté(s).

Les exemples (3) nous permettent de signaler que l'argument de difficulté (et donc de difficile) a une interprétation d'évènement au sens large. En d'autres termes, il décrit une situation mettant en jeu un processus ou un état et ses participants. En tant que tel, il s'inscrit dans l'une des quatre classes aspectuelles définies par Vendler (1967) (état, activité, accomplissement, achèvement). Il s'agit cependant de la seule contrainte sur cet argument, puisque tout phénomène susceptible de provoquer une ou des difficultés peut apparaitre comme sujet ou complément (cf. (3)). Pour cette raison, il nous a semblé intéressant, en relation avec d'autres travaux que nous avons menés sur les noms désadjectivaux (Knittel, 2015a et b), d'entreprendre l'étude du nom difficultés, particulièrement fréquent dans ce contexte spécialisé du CRBO (cf. 3.1).

Par ailleurs, nous portons notre intérêt sur la terminologie propre au domaine de l'orthophonie, et plus spécifiquement sur les éléments 
linguistiques (syntaxiques comme sémantiques) susceptibles de révéler les représentations de ce qu'est la réalité du patient pour l'orthophoniste (Brin-Henry, 2014). En effet, lorsqu'il essaie de situer les troubles du langage observés dans le continuum conceptuel partant du langage ordinaire ou atypique efficient jusqu'au langage pathologique, l'orthophoniste est confronté à une double considération qui fait partie intégrante de la démarche diagnostique. Il considère ainsi à la fois le fond («Où se situe la norme? Quelles sont les limites des pathologies?») et la forme («Est-ce bien une dyslexie? Ce tableau m'indique-t-il autre chose?»), dans une perspective onomasiologique - partant du concept jusqu'au choix du terme - et sémasiologique, du terme jusqu'aux concepts des pathologies. Nous avons choisi de nous consacrer en premier lieu à la démarche d'étiquetage des pathologies observées, qui s'opère au moyen de libellés variables. Nous présupposons que les variantes de ces libellés auxquelles recourent les orthophonistes en rédigeant leurs comptes rendus révèlent par des routines chez ces spécialistes des conceptions particulières des pathologies du langage, qui évoluent dans le temps. À ce titre, l'analyse conjointe des variations proposées par ces professionnels sous les angles syntactico-sémantique et terminoontologique enrichit la réflexion sur le lien entre la terminologie et les concepts sous-jacents relatifs à la pathologie.

Nous avons d'ores et déjà observé (Brin-Henry, 2011b) :

- une inconstance diachronique des termes. Par exemple, le terme alalie créé par Lordat en 1863 a été progressivement supplanté par aphasie (créé en 1864 par Armand Trousseau);

- une inconstante synchronique des termes. Ainsi, le terme sigmatisme interdental alterne avec zozotement dans les comptes rendus;

- une fréquence élevée d'apparition des noms difficultés et troubles;

- une utilisation exclusive de l'une ou l'autre parmi deux formes de termes diagnostiques : des locutions et composés d'une part (retard de langage, dyslexie), ou des syntagmes ayant des caractéristiques plus ou moins collocatives d'autre part (troubles de la compréhension des énoncés complexes).

À plus long terme, cette étude s'inscrit dans nos travaux sur la pose du diagnostic de langage pathologique par les orthophonistes, et contribue à la discussion sur les classifications des troubles du langage 
dans une perspective terminologique et sémiotique (Reilly, Bishop \& Trombin, 2014; Wirotius, 2012; Brin-Henry, 2011a).

\subsection{Le nom difficulté(s) dans les CRBO}

Les CRBO sont la trace écrite par les orthophonistes à la suite d'un bilan au cours duquel sont mesurés et évalués les potentialités et les déficits des patients. Ils sont destinés aux professionnels de santé (notamment les médecins prescripteurs du bilan). Ils bénéficient depuis 2003 d'une architecture rédactionnelle par convention avec la sécurité sociale ${ }^{2}$. Ils mentionnent généralement les tâches demandées (tests standardisés), les résultats obtenus, et comportent entre autres un diagnostic («terme diagnostique») et un plan thérapeutique. Il s'agit d'un genre discursif propre à ces professionnels. La constitution d'un corpus et l'exploration de ces textes permettent d'aborder la question des représentations sousjacentes des pathologies et la façon dont la terminologie employée dans le CRBO les révèle.

La présente étude porte sur un ensemble de 1230 occurrences du nom difficulté( $s$ ) représentant l'ensemble des usages de cet item dans un corpus de 436 CRBO.

\section{Méthodologie}

\subsection{Appui sur un corpus}

Ce corpus ${ }^{3}$ écrit d'environ 280000 mots a été constitué en 2007 dans le cadre d'une thèse en sciences du langage, à partir de 436 CRBO anonymisés recueillis auprès de plus de 80 orthophonistes français (Brin-Henry, 2011b). La fréquence d'apparition des mots a été évaluée (KwikList) grâce au concordancier AntConc ${ }^{4}$ et a montré l'occurrence remarquable du nom difficulté(s) :

- difficulté : 226 occurrences dans le corpus (140e rang sur 12607);

- difficultés : 1004 occurrences (39ª rang).

2. JORF du 27 février 2003, page 3499, texte $n^{\circ} 27$, Avenant à la convention nationale des orthophonistes, NOR: SANS0320601X [en ligne], <www.legi france.gouv.fr/eli/avenant/2003/2/27/SANS0320601X/jo/texte>.

3. Ce corpus est confidentiel en raison du secret professionnel imposé par le Code de la santé publique (article L 1110-4).

4. Cf. <www.laurenceanthony.net/software.html>. 
La fréquence d'emploi de cet item le place immédiatement parmi les mots-clés du domaine, bien qu'il ne s'agisse pas à proprement parler d'un terme spécialisé. Une étude complémentaire consacrée à ce mot-clé nous a permis de repérer les concordances dans AntConc au moyen d'une expression régulière (contexte : 2 à gauche / 4 à droite). L'objectif de cette discussion est de tester l'hypothèse selon laquelle les représentations des pathologies du langage des orthophonistes seraient objectivables à partir de patrons lexico-sémantiques propres à ces professionnels, lisibles au travers de l'examen des variantes utilisées dans le corpus. Ces variantes portent parfois une valeur terminologique diagnostique comme nous le verrons en 5.2 (iii).

\subsection{Méthodologie de l'étude}

Dans un premier temps, nous nous sommes concentrées sur le contexte droit, plus varié que le contexte gauche. Nous avons procédé à un repérage des éléments qui apparaissent après le nom difficultés (voir section 4), en nous intéressant d'abord au type syntaxique de ces constituants; nous avons ensuite procédé à leur catégorisation sémantique en nous interrogeant sur le type d'information véhiculé par les éléments présents dans l'environnement du nom difficultés. Notre hypothèse étant que la syntaxe et la sémantique se reflètent mutuellement, nous supposons que des valeurs sémantiques différentes seront encodées au moyen de structures syntaxiques différentes. Nous situons donc ce travail à l'interface syntaxe/sémantique. Nous pensons en effet que ce niveau de description constitue l'étape préliminaire au repérage des routines discursives.

\section{L'étiquetage du corpus}

L'étiquetage de notre corpus a été réalisé manuellement dans un tableur créé à partir du fichier .txt du concordancier. La première colonne du tableau fournit les contextes gauches du lemme difficulté, la deuxième l'ensemble des occurrences du lemme, la troisième le contexte droit dans l'ordre alphabétique. C'est ce dernier qui fait l'objet de notre attention, et que nous avons trié selon des critères syntaxiques (cf. section 4.1), puis sémantiques (cf. section 4.2). 


\section{1. Étiquetage syntaxique}

Le premier stade de l'étiquetage a été de répertorier les catégories présentes dans le contexte droit du nom difficulté( $s$ ) et pouvant être analysées comme ses dépendances syntaxiques. De ce fait, nous avons pour l'instant exclu l'étude de la combinatoire avec des verbes supports (cf. section 2.1), situés en contexte gauche et dont le nom est une dépendance. Bien que ces constructions soient fréquentes, comme nous le verrons dans les exemples qui vont suivre, nous leur réserverons une étude ultérieure.

Nous avons recensé trois classes de constituants particulièrement fréquents.

\section{(i) Syntagmes prépositionnels}

Les syntagmes prépositionnels $(\mathrm{P}+\mathrm{SN}$, dorénavant $\mathrm{SP})$ recensés sont introduits par des prépositions simples (5) ou par des locutions prépositives (6) :

a. $[\mathrm{X}]$ consulte pour des difficultés [en lecture $]_{\mathrm{SP}}$

b. [X] est une enfant de 4 ans qui éprouve des difficultés [d'expression] $]_{\mathrm{SP}}$.

(6)

a. Il y a quelques difficultés [au niveau de la parole et de l'articulation $]_{\mathrm{SP}}$

b. Mais des difficultés encore [face à des phrases $]_{\mathrm{SP}}$.

Les SP des exemples (5), qui comportent un nom déverbal, peuvent être caractérisés comme des arguments du nom difficultés. En effet, ces SP sont paraphrasables par des subordonnées infinitives susceptibles d'apparaitre tant avec le nom qu'avec l'adjectif, dont elles peuvent être sujet (lire/s'exprimer est difficile) ou complément (il est difficile de lire/s'exprimer), comme nous l'avons précisé en 2.1.

Parmi les prépositions simples, on peut relever la présence de de, qui introduit fréquemment, en concurrence avec chez et pour, moins fréquents (une occurrence chacun), la référence à l'individu présentant les difficultés (voir 4.2) :

(7)

a. les parents ne se rendent pas compte des difficultés [de Théo $]_{\mathrm{SP}}$

b. son institutrice qui a remarqué des difficultés [chez cette enfant $]_{\mathrm{SP}}$

c. les épreuves suivantes présentant le même type de difficulté [pour lui $]_{\mathrm{SP}}$.

Ce type de SP, qui instancie le rôle de Siège (cf. section 4.2 (i) ci-dessous) peut être considéré comme un argument que le nom diffi- 
culté(s) hérite de son adjectif-base. Il peut en effet apparaitre sous forme de clitique lorsque l'adjectif est employé comme attribut (cf. il lui est difficile de passer cette épreuve), ce qui caractérise certains arguments internes, notamment de type Experiencer ou Siège, selon Tayalati et Van Peteghem (2009).

\section{(ii) Subordonnées}

Les subordonnées (Sub) dépendantes du nom difficultés sont de natures diverses. Il peut s'agir d'infinitives $(8 \mathrm{a})$ ou de subordonnées à temps fini (8b), parmi lesquelles des relatives (8c).

a. ses difficultés [à combiner rapidement les sons entre eux $]_{\text {Sub }}$

b. mais encore des difficultés [lorsqu'il y a une séquence d'actions à réaliser $]_{\text {Sub }}$

c. ce qui montre les nombreuses difficultés [qu'il éprouve dans l'analyse de la chaine parlée] $]_{\text {SubRel }}$.

Au même titre que les SP en de/chez/pour, les subordonnées infinitives (8a) sont des arguments hérités de l'adjectif correspondant.

\section{(iii) Adjectifs}

Les adjectifs (Adj) sont particulièrement fréquents dans l'environnement du nom difficulté(s). Il faut cependant distinguer deux classes. D'une part, certains adjectifs se caractérisent comme taxinomiques (McNally \& Boleda, 2004; Knittel, 2005). Sémantiquement, ils indiquent le type de difficulté rencontré. Syntaxiquement, ils sont toujours adjacents à droite du nom, et ne sont ni prédicatifs, ni gradables $^{5}$ (9). Ils se comportent donc comme des adjectifs relationnels, sans toutefois être dénominaux (voir sur ce point Bosredon, 1988).

5. On peut ainsi comparer (i-ii) à (iii-iv) :

(i) * Cette difficulté est gnosique AdjTax

(ii) *Une difficulté très gnosique AdjTax

(iii) Cette difficulté est fréquente Adjoual $_{\text {. }}$

(iv) Une difficulté très fréquente AdjQual $_{\text {. }}$

Notons que l'emploi prédicatif (i.e. attribut) d'un adjectif taxinomique est plus acceptable si l'adjectif est introduit par de type; de la même manière, l'adverbe typiquement peut être employé pour spécifier cette classe d'adjectifs :

(v) Cette difficulté est \{de type / typiquement\} gnosique AdjTax $_{\text {. }}$

(vi) *Cette difficulté est $\{$ de type/ typiquement $\}$ fréquente ${ }_{\text {Adjoul }}$. 
Dans ce cas, l'adjectif est fréquemment la seule dépendance postnominale présente :

(9)

a. Pour pallier aux difficultés gnosiques ${ }_{\mathrm{Adj} T \mathrm{Tax}}$ elle essaye de décoder des indices.

b. S. ne présente pas de grosses difficultés articulatoires ${ }_{\mathrm{AdjT} T \mathrm{~T}}$.

À l'inverse, les adjectifs qualificatifs sont généralement cooccurrents avec une autre dépendance instanciant le complément de l'adjectif :

a. Des difficultés importantes ${ }_{\mathrm{Adj} \text { Qual }}$ [au niveau du langage écrit $]_{\mathrm{SP}}$

b. Lecture de nombres : difficulté majeure $\mathrm{Adj}_{\text {Qjual }}[\text { au delà de la dizaine }]_{\mathrm{SP}}$.

Comme le montrent les exemples ci-dessus, notamment (9b-10b), le nom difficulté(s) peut être suivi de plusieurs dépendances; ceci est confirmé par les exemples suivants, qui montrent une double complémentation, de type $[\mathrm{de}+\mathrm{SN}][\mathrm{a}+\mathrm{Sub}]$ :

a. les difficultés [d'Adrien $]_{\mathrm{SP}}[\text { à effectuer des soustractions }]_{\text {Sub }}$

b. les difficultés [de ce patient $]_{\mathrm{SP}}[\mathrm{à} \mathrm{les} \mathrm{conserver} \mathrm{ou} \mathrm{les} \mathrm{reconduire}]_{\mathrm{Su}}$.

Ce premier recensement montre que difficulté( $(s)$ présente à la fois des dépendances des noms en général (Relatives, SP, Adjectifs, etc.), et d'autres instanciant ses arguments, comme certains SP (7), les subordonnées infinitives (8a), et les noms déverbaux (5b), ce qui constitue une spécificité due à son caractère désadjectival. La richesse et la variété des dépendances de ce nom en font donc un élément particulièrement intéressant à étudier, notamment en comparaison avec d'autres items de sens relativement proche (cf. section 5.2).

\subsection{Typologie sémantique}

À la suite du classement syntaxique, nous avons établi un classement des contextes droits selon le type d'information explicité dans la dépendance. Nous avons considéré que ces contextes permettent d'apporter des informations sur l'identité des difficultés qui s'expriment chez le patient et qui sont susceptibles d'être identifiées par l'orthophoniste.

\section{(i) Informations relatives au patient}

La référence au patient qui présente la/les difficultés est prioritairement exprimée au moyen d'un SP en de (cf. (7)), qui peut alterner avec 
un déterminant possessif (cf. ses difficultés). En termes sémantiques, ce type de dépendance instancie le rôle de Siège, que l'on peut définir comme une entité animée ressentant un sentiment ou se trouvant dans un certain état psychologique.

\section{(ii) Informations relatives à l'environnement}

Ce type de dépendance, étiqueté Env., permet d'exprimer le lieu où se produisent les difficultés.

a. mais connait d'importantes difficultés [scolaires $]_{\mathrm{Env}}$ depuis le Cours Préparatoire.

b. bilan réalisé ne permet donc pas d'expliquer les difficultés [au collège $]_{\text {Env. }}$ rapportées par A et sa mère.

c. malgré d'importantes difficultés [familiales] $]_{\mathrm{Env}}$

\section{(iii) Informations relatives au temps}

Dans ce domaine, les informations, étiquetées Temp., concernent la durée (13a), la fréquence (13b) et l'ancrage temporel (13c) :

a. des difficultés [persistantes $]_{\text {Temp. }}$ en lecture

b. qui reste conscient de ses difficultés [au quotidien] $]_{\text {Temp. }}$

c. difficultés [au cours du premier trimestre $]_{\text {Temp. }}$

\section{(iv) Informations relatives à l'incidence et à l'intensité}

Ce type d'information est majoritairement spécifié par des adjectifs qualificatifs. Dans 31 cas, c'est le caractère important de la difficulté qui est signalé (adjectifs important, majeur, etc., cf. (10-14b)). L'incidence de la difficulté est précisée dans 12 cas (par exemple (14a)).

a. ceci malgré ses difficultés [encore très présentes $]_{\text {Int. }}$.

b. des difficultés [prépondérantes] $]_{\text {Int. }}$ en orthographe syntaxique

c. mise en évidence de difficulté [importante et significative $]_{\text {Int. }}$.

d. ils s'inscrivent dans un tableau de difficultés [plus globales] $]_{\text {Int }}$

On notera que les informations des classes (iii) et (iv) sont majoritairement exprimées par des adjectifs qualificatifs.

(v) Informations relatives à la pratique de l'orthophonie (situation de bilan)

Ces informations, notées Ortho., sont spécifiques au domaine de référence étudié. Les dépendances spécifient les tâches du bilan standardisé 
au cours desquelles l'orthophoniste a observé les difficultés. Leur formulation est conditionnée par la situation particulière de séances d'orthophonie. Plutôt que les causes des difficultés, il nous semble que le contexte droit des occurrences révèle la façon dont la difficulté se manifeste ${ }^{6}$.

a. XXX a beaucoup de difficultés [pour les tâches de classification] $]_{\text {Ortho. }}$

b. Des difficultés [attentionnelles $]_{\text {Ortho. }}$ altèrent la réalisation des épreuves.

c. Il semble parfois éprouver des difficultés [à se concentrer] $]_{\text {Ortho. }}$

d. Elle a des difficultés [de compréhension du vocabulaire $]_{\text {Ortho. }}$

\section{Résultats}

Suite à l'étiquetage syntaxique (4.1) et à la classification sémantique (4.2) des dépendances, nous avons croisé les deux critères de classement, afin d'examiner leurs interactions. Nous présentons ci-dessous nos résultats selon l'angle structural (5.1) puis en interrogeant les variations en ce qu'elles révèlent les représentations des orthophonistes quant aux pathologies du langage (5.2).

\subsection{Classement structural}

\section{(i) Adjectifs}

Le moyen formel utilisé de manière prépondérante pour caractériser le terme difficulté(s) est l'emploi d'adjectifs. Comme on peut s'y attendre (cf. classes (iv) vs (v) ci-dessus)), les adjectifs en question ne sont pas employés en tant que qualificatifs, mais comme des adjectifs taxinomiques (cf. 4.1 (iii)), c'est-à-dire pour exprimer des sousclasses du nom qu'ils modifient. À ce titre, et notamment lorsqu'ils sont d'occurrence fréquente, on peut considérer certains de ces syntagmes (cf. (16c)) comme des collocations, selon l'approche statistique de Granger et Paquot (2008), d'autant plus lorsqu'ils sont utilisés comme des termes diagnostiques.

6. Si l'on considère que les tâches demandées servent au praticien pour repérer les déficiences des patients, on pourrait considérer que ce type de dépendance renvoie à un instrument, si l'on admet que cette classe peut renvoyer à des abstraits. 
(16)

a. Ses difficultés lexicales ${ }_{\mathrm{AdjTax}}$ et syntaxiques AdjTax $_{\text {Tax }}$

b. On relève par contre une difficulté articulatoire AdjTax $_{\text {. }}$

c. Quelques difficultés mnésiques ${ }_{\text {Adjax }}$ (moindre capacité de fixation).

Sur le plan morphologique, on note que ces adjectifs peuvent être dénominaux ((16a) : lexical sur lexique; syntaxique sur syntaxe); ils sont alors traditionnellement appelés relationnels (Bally, 1965 ; Roché, 2006; Fradin \& Kerleroux, 2003, 2009; voir également Maniez, 2009), comme nous l'avons spécifié ci-dessus. Ils peuvent également être déverbaux ((16b) : articulatoire sur articuler). Le cas de mnésique (16c) révèle l'emploi de formes dites savantes, fréquent dans les lexiques spécialisés.

(ii) SP

Les $\mathrm{SP}(\mathrm{P}+\mathrm{SN})$ les plus fréquents pour introduire des informations relatives à la pratique de l'orthophonie sont ceux qui sont introduits par en et par de; nos données indiquent en outre une spécialisation selon la préposition.

- difficultés en est utilisé pour décrire des troubles relatifs au domaine scolaire, et pour la plupart d'ordre linguistique :

difficultés en compréhension écrite / conjugaison / langage écrit / transcription / lecture et orthographe / calcul /...

En est fréquemment suivi d'un nom nu (i.e. ni quantifié, ni modifié) comme dans la langue générale, ou d'une collocation (Tutin \& Grossman, 2002).

- Au contraire, difficultés de (lorsqu'il n'introduit pas le Siège, cf. exemples (7) et 4.2 (i)) introduit souvent des séquences plus longues, dans lesquelles le premier nom, généralement déverbal, est quasiment toujours associé à un complément $(\mathrm{Ct})$ :

a. difficultés [de rétention $\left.{ }_{N}\left[d^{\prime} \text { 'informations verbales }\right]_{\mathrm{Ct}}\right]_{\mathrm{SP}}$

b. difficultés [d'analyse $\left.{ }_{\mathrm{N}}[\text { de la chaîne graphémique }]_{\mathrm{Ct}}\right]_{\mathrm{SP}}$

c. difficultés [d'accès $\left.\mathrm{N}_{\mathrm{N}}[\mathrm{au} \text { langage élaboré }]_{\mathrm{C}}\right]_{\mathrm{SP}}$.

Ces exemples, notamment (18a) et (18c), illustrent bien la recherche de précision qui caractérise les orthophonistes lorsqu'ils déterminent l'identité de la difficulté observée chez la personne qui consulte. 


\section{(iii) Subordonnées}

Les subordonnées exprimant des informations relatives à la pratique de l'orthophonie sont des infinitives introduites par $\grave{a}$; cette préposition alterne avec de (Knittel \& Koehl, 2013), comme nous le verrons ci-dessous (5.2 (i)).

(19)

a. On note ici une légère difficulté [à traiter des informations écrites plus complexes $]_{\text {Sub }}$

b. ses difficultés [à combiner rapidement les sons entre eux $]_{\text {Sub }}$.

\subsection{Fonction des variations}

Nous avons précisé plus haut que l'approche retenue ici, entre sémantique et syntaxe, nous permet d'examiner les formes relevées en tant que variantes terminologiques.

Nous pensons que les praticiens ont été et sont créateurs de certaines de ces variantes terminologiques (formes linguistiques différentes des termes, les variations étant les phénomènes menant à la production des diverses variantes) dans les routines scripturales. Ainsi on différenciera «troubles articulatoires » et «troubles d'articulation», «dyslexie» et «troubles de la compréhension des énoncés» (voir Desmet, 2006). En adoptant des variantes, l'orthophoniste tente parfois de combler un vide conceptuel (une entité ou un sous-groupe pathologique qui jusqu'à présent n'était pas identifié comme tel) que ces syntagmes permettent d'étiqueter. Ces créations dérivent parfois - tant par généralisation que dans une démarche d'opposition - de propositions institutionnelles classificatoires (ex. du DSM5 ${ }^{7}$ ), ou règlementaires $\left(\mathrm{NGAP}^{8}\right)$. Elles suivent ainsi les courants de pensée actuels, qui s'imprègnent progressivement d'une conception plus sociétale de la pathologie (voir 5.2 (iii)).

Dans d'autres cas, les variantes résultent d'une procédure onomasiologique individuelle, mais qui peut s'étendre au groupe de professionnels dans son ensemble. Nous pensons que le choix terminologique

7. Le Diagnostic and Statistical Manual publié par l'American Psychological Association est un ouvrage de référence internationale (quoique très critiqué) qui classifie et catégorise des critères diagnostiques et des recherches statistiques de troubles mentaux spécifiques depuis les années 1980. La 5édition est parue en 2013 .

8. Nomenclature générale des actes professionnels. 
(recours à la variation) peut se décrire selon plusieurs patrons, répondant à des questions du type «Quels sont les problèmes?», et «Comment / Où sont les problèmes?» portant des structures syntaxiques spécifiques comme l'illustre en exemple le tableau 1 ci-dessous.

\begin{tabular}{|l|l|l|l|l|}
\hline & $\begin{array}{l}\text { Construction } \\
\text { prépondérante }\end{array}$ & $\begin{array}{l}\text { Ce qu'elles } \\
\text { expriment }\end{array}$ & $\begin{array}{l}\text { Opposition } \\
\text { signifiante }\end{array}$ & Spécification \\
\hline difficultés & + Adj.Tax. & $\begin{array}{l}\text { Domaine dont } \\
\text { relève la difficulté } \\
5 \text { classes } \\
\text { nosologiques }\end{array}$ & $\begin{array}{l}\text { Circonstances de } \\
\text { la difficulté }: \text { de }+\mathrm{N} \\
\text { vs à+V } \\
\text { difficulté d'accès vs } \\
\text { difficulté à accéder }\end{array}$ & $\begin{array}{l}\text { Adj.Qual. } \\
\text { autres SP } \\
\text { Sub. }\end{array}$ \\
\hline
\end{tabular}

Tableau 1. - Proposition de classement des patrons de «difficultés».

Nous avons d'abord observé deux patrons (qui acceptent certaines combinaisons) dont la fonction est d'expliciter l'identification (I) de la difficulté, qui répondent à la question «Quels sont les problèmes?» (Quoi).

L'orthophoniste souhaitant exprimer le domaine de la difficulté (D) utilise préférentiellement les motifs difficultés +Adj.Tax, et difficultés + en N. Pour préciser les circonstances (C) dans lesquelles se sont manifestées les difficultés, difficultés + à $\mathrm{V}$ et difficultés + de $\mathrm{N}$ sont préférés.

S'il s'agit de spécifier (S) la difficulté en évoquant l'environnement, l'ancrage temporel, l'incidence et l'intensité, ce sont difficultés + Adj. Qual, difficultés + SP (autres que ceux en de et en), et difficultés + Sub. qui sont privilégiés.

Cette seconde série de patrons répondent à aux questions «Comment/Où sont les problèmes?»(Où). Ces patrons admettent certaines combinaisons de type $\mathrm{D}+\mathrm{S}$ ((24a) des difficultés orthographiques évidentes), $\mathrm{C}+\mathrm{S}$ (des difficultés importantes d'acquisition du langage écrit), ou encore $\mathrm{S}+\mathrm{S}((10 \mathrm{a})$ des difficultés importantes au niveau du langage écrit).

Ainsi, certaines oppositions et variations nous paraissent révéler des conceptions différentes des pathologies du langage par l'utilisation d'une ou l'autre formulation (voir François, 2010 pour ce type d'approche). Nous en présenterons trois types. 
(i) Opposition des formes : difficultés de+N (SP) vs difficultés à+V (Sub)

Sur le plan formel, le type de difficulté (cf. section 2.1) peut être introduit tant par une subordonnée en $\grave{a}+$ Verbe à l'infinitif que par un $\mathrm{SP}$ en de suivi du nom déverbal correspondant à l'infinitive.

a. une difficulté [à accéder à la pensée abstraite]

b. une difficulté [d'accès à la signification de certaines tournures].

a. une difficulté [à lire les syllabes complexes]

b. des difficultés [de lecture et d'orthographe].

a. Rappelons la difficulté [à mémoriser des séquences de sons complexes]

b. difficultés [de mémorisation à partir de phrases].

Dans ce type d'alternance, les SP en $d e+\mathrm{SN}$ permettent à l'orthophoniste de transmettre une idée de stabilité (20b) ou de généralisation, de globalité (21b;22b) de la/des difficulté(s). À l'inverse, l'utilisation de Subordonnées en à $+\mathrm{V}$ Infinitif traduit l'idée que la difficulté est observée dans une activité en cours (22a). C'est alors l'aspect actif du processus qui est analysé comme pathologique.

\section{(ii) Variation de l'adjectif taxinomique}

Les 138 occurrences d'adjectifs taxinomiques (4.1 (iii)) repérés dans le corpus ont pu être regroupées en cinq domaines, présentés ci-dessous.

(23) domaine cognitif (53 occurrences sur 138) :

a. les difficultés attentionnelles ${ }_{\mathrm{AdjTax}}$ sont manifestes

b. des difficultés exécutives ${ }_{\mathrm{Adj} T a x}$ de récupération en mémoire

c. afin de voir si A vraiment des difficultés intellectuelles $\mathrm{AdjTax}_{\text {. }}$.

(24) domaine linguistique (44/138) :

a. montre des difficultés orthographiques ${ }_{\text {AdjTax }}$ évidentes

b. L'enfant essaie de compenser ses difficultés expressives ${ }_{\text {AdjTax }}$ en recourant aux mimes

c. les difficultés phonétiques ${ }_{\mathrm{Adj} T a x}$ sont ici prédominantes.

(25) domaine socio-relationnel (19/138) :

a. malgré d'importantes difficultés familiales ${ }_{\text {AdjTax }}$

b. entrainent $[\ldots]$ des difficultés psychologiques ${ }_{\mathrm{AdjTax}}$

c. des difficultés relationnelles AdjTax $_{\text {à l'école. }}$ 
(26) domaine physique (13/138) :

a. ses difficultés auditives ${ }_{\text {AdjTax }}$ sont stables depuis plusieurs années

b. il a tendance à garder la bouche ouverte (difficulté $\mathrm{ORL}_{\text {AdjTax }}$ )

c. les difficultés sont en relation avec des difficultés perceptives ${ }_{\mathrm{Adj} T a x}$.

(27) domaine moteur (9/138) :

a. on note par ailleurs des difficultés graphiques ${ }_{\mathrm{AdjTax}}$ importantes,

b. pas de troubles associés ni de difficultés motrices ${ }_{\text {AdjTax }}$

c. on note des difficultés psychomotrices ${ }_{\text {AdjTax }}$.

On notera la prédominance des domaines cognitif et linguistique dans la sélection de ces adjectifs, ce qui s'explique par l'ancrage médical historique de la discipline. Le domaine socio-relationnel, très présent également, montre l'intérêt des professionnels pour la relation, et révèle l'introduction progressive du modèle social du handicap (Fougeyrollas, Cloutier, Bergeron, Cote \& Michel, 1998) qui se généralise dans le champ de la santé depuis les publications de l'Organisation mondiale de la santé comme la $\mathrm{CIF}^{9}$. Dans ce modèle, c'est le regard et la noninclusion de la personne dans une société éminemment normative qui crée le handicap.

\section{(iii) Variation connotative du nom}

Dans le corpus étudié, le nom difficulté(s) apparait très fréquemment en tête des syntagmes utilisés comme variantes, tant dans le texte que dans les termes diagnostiques. Sur le plan syntaxique, ces syntagmes sont nettement moins figés que les autres termes diagnostiques du corpus, qui relèvent de l'unité lexicale (bégaiement) ou de la collocation (dysphasie visuo-constructive). Sémantiquement parlant, ils rendent compte d'un dysfonctionnement dans un système (par exemple système scriptural, verbal). Les syntagmes comprenant le nom difficultés font, parmi d'autres, référence à un processus dynamique déviant chez le patient. Ce phénomène se rencontre également avec d'autres noms (désadjectivaux ou déverbaux) dont la répartition fréquentielle est relativement peu homogène. Ainsi, parmi les 558 termes diagnostiques présents dans les comptes rendus examinés, 18 (dont difficulté(s)) sont employés pour décrire la pathologie sur un plan fonctionnel. Ceci est détaillé dans le tableau 2 ci-dessous.

9. Classification internationale du fonctionnement, du handicap et de la santé élaborée par l'Organisation mondiale de la santé (OMS) en 2001. 


\begin{tabular}{|l|c|}
\hline Étiquettes de lignes & Nombre \\
\hline ALTÉRATION & 2 \\
\hline ATTEINTE & 8 \\
\hline CARENCE & 1 \\
\hline DÉFAILLANCE & 1 \\
\hline DÉFAUT & 2 \\
\hline DÉFICIT(S) & 13 \\
\hline DÉVIANCES & 1 \\
\hline DIFFICULTÉ(S) & 53 \\
\hline DYSFONCTIONNEMENT & 2 \\
\hline FAIBLESSE(S) & 4 \\
\hline PATHOLOGIE(S) & 7 \\
\hline PAUVRETÉ & 1 \\
\hline PROBLÈMES & 1 \\
\hline RETARD & 30 \\
\hline SÉMIOLOGIE & 3 \\
\hline SÉQUELLE(S) & 15 \\
\hline TABLEAU & 2 \\
\hline TROUBLE(S) & $\mathbf{2 9 8}$ \\
\hline Total général & \\
\hline
\end{tabular}

Tableau 2. - Répartition des termes diagnostiques révélant un déficit d'activation d'un système.

Selon nous, l'item difficultés apparait, au sein des termes diagnostiques, dans un syntagme ou une glose pour décrire des symptômes, en opposition à des locutions ou des composés dont nous pensons qu'ils signalent un déficit du système tout entier. Par exemple, alors que le terme difficultés de compréhension des phrases complexes fait état d'un problème d'utilisation de la fonction de lecture, celui de dyslexie évoque une pathologie intrinsèque à la personne, une atteinte d'une structure ou d'un système. De même, dans le discours orthophonique coexistent difficultés de structuration phonologique et retard de parole qui ne peuvent être superposables, le premier évoquant une mise en place compliquée de l'activation du système permettant l'articulation correcte du français, le second alléguant d'une atteinte de ce même système. Précisons que le système (ou la structure) dont nous parlons ici peut être anatomique ou physiologique, mais elle est principalement symbolique. 
Cette opposition entre troubles de la structure (6a, 15c et 16a) et troubles dans l'utilisation de la structure (9b, 18a) se retrouve dans l'ensemble du corpus et devra bénéficier d'un examen complémentaire. En particulier, une étude contrastive, à la manière de Veniard (2008), entre difficulté(s) et trouble(s) est envisagée afin d'examiner ce qui sous-tend l'emploi de l'un ou l'autre de ces termes, particulièrement dans le sousgroupe formé par les termes diagnostiques (cf 5.2 (ii)). Nous pensons en effet que l'utilisation de trouble $(s)$ renvoie à une représentation plus «médicale» de l'atteinte que celle de difficulté(s).

\section{Bilan et perspectives}

Comme nous venons de le montrer, l'étude sémantico-syntaxique des occurrences du nom difficulté(s) dans un corpus de comptes rendus de bilan orthophonique révèle des utilisations variées de cet item. Sur le plan syntaxique, ce désadjectival est associé à des syntagmes prépositionnels, des adjectifs qualificatifs et taxinomiques, ainsi qu'à des structures comportant des verbes (subordonnées). Sur le plan sémantique, il véhicule des informations relatives à la situation de la personne dans son environnement, et dans sa rencontre avec un(e) orthophoniste. L'examen conjoint des contraintes syntaxiques et sémantiques montre d'une part la prédominance de structures longues évoquant une taxinomie complexe, d'autre part l'importance de structures verbales indiquant un processus dynamique en cours, et enfin l'utilisation d'adjectifs établissant l'interpénétration de considérations médicales et relationnelles.

L'examen des résultats sous l'angle de l'analyse des routines discursives fait émerger l'existence de deux types de patrons distincts, qui identifient ou spécifient les problèmes selon l'axe du «Quoi» et l'axe du «Où». Notons que, selon nous, la référence au patient ne constitue pas réellement un patron. En effet, il est rarement mentionné en contexte droit, mais apparait plutôt de façon implicite ou dans le contexte gauche comme le constatent également Lövestam et coll. (2015) sur un corpus rédigé par des diététiciennes.

À ce stade de notre travail, plusieurs perspectives s'offrent à nous. D'une part, l'étude des contextes gauches, et en particulier des verbessupports (Gross, 1981) (manifester/éprouver/connaitre / avoir / montrer / ressentir \{une/des\} difficultés, voir Anscombre, 1995) pourrait certainement enrichir l'étude des représentations des pathologies du 
langage chez les orthophonistes. D'autre part, une étude contrastive des environnements du nom difficulté( $s$ ) dans la langue générale permettrait de mieux cerner les spécificités de cet item dans la terminologie orthophonique. Enfin, comme nous l'avons évoqué en 5.2 (iii), la comparaison des emplois de difficulté(s) face aux autres termes signalant une atteinte du fonctionnement d'un système nous parait une autre piste fructueuse.

L'examen conjoint des contraintes syntaxiques et des variations sémantiques nous semble une méthodologie prometteuse, pour plusieurs raisons. Elle permet d'abord d'enrichir notre connaissance des processus de création terminologique. Ensuite, sur un plan disciplinaire, ces considérations permettent de porter un regard nouveau sur les pathologies. Enfin, l'entrée linguistique dans l'examen du discours sur la pathologie du langage dans les écrits professionnels et scientifiques, en offrant une perspective terminologique novatrice dans ce domaine, permet de dépasser les clivages idéologiques et théoriques en orthophonie.

\section{RÉFÉRENCES BIBLIOGRAPHIQUES}

Anscombre, Jean-Claude. (1995). Morphologie et représentation évènementielle : le cas des noms de sentiment et d'attitude. Langue française, 105 , 40-54.

BALLY, Charles. (1965). Linguistique générale et linguistique française (4e éd.). Berne : A. Francke.

BosRedon, Alain. (1988). Un adjectif de trop : l'adjectif de relation. L'information grammaticale, 37, 3-7.

BRIN-HenRY, Frédérique. (2011a). Quelle classification des troubles du langage écrit pour les orthophonistes? Dans E. Lederlé (dir.), Les troubles du langage écrit : regards croisés. Isbergues : Ortho Édition.

BRIN-HeNRY, Frédérique. (2011b). La terminologie crée-t-elle la pathologie? Le cas de la pratique clinique de la pose du diagnostic orthophonique (Thèse de doctorat en sciences du langage, Université Nancy 2). Disponible en ligne sur <http://tel.archives-ouvertes.fr/tel-00655952/> (consulté le 23 mars 2015).

Brin-Henry, Frédérique. (2014). Using Corpus-Based Analyses in Specialised Paramedical French. Revue française de linguistique appliquée («Langues de spécialité : problèmes et méthodes»), 19(1), 103-115.

Condamines, Anne. (2007). L'interprétation en sémantique de corpus : le cas de la construction de terminologies. Revue française de linguistique appliquée, 12(1), 39-52. 
DESMET, Isabelle. (2006). Variabilité et variation en terminologie et langues spécialisées : discours, textes et contextes. Mots, termes et contextes, 235-247.

Fougeyrollas, Patrick, Cloutier, René, Bergeron, Hélène, Cote, Jacques \& Michel, Ginette. (1999). Classification québécoise : processus de production du handicap. Québec : Réseau international sur le processus de production du handicap (RIPPH)/SCCIDIH.

Fradin, Bernard \& Kerleroux, Françoise. (2003). Quelles bases pour les procédés de la morphologie constructionnelle? Dans B. Fradin, G. Dal, N. Hathout, F. Kerleroux, M. Plénat \& M. Roché (dir.), Silexicales 3 : les unités morphologiques (p. 76-84). Villeneuve-d'Ascq : Presses universitaires du Septentrion.

Fradin, Bernard \& Kerleroux, Françoise. (2009). L'identité lexémique. Dans B. Fradin, F. Kerleroux \& M. Plénat (dir.), Aperçus de morphologie du français (p. 85-104). Saint-Denis : Presses universitaires de Vincennes.

FRAnÇOIS, Jacques. (2010). Le repérage des segments répétés et la polysémie lexicale (à propos des cooccurrences de $\mathrm{FORCE}(\mathrm{S})$ dans trois corpus journalistiques). Revue de Sémantique et Pragmatique, 27, 11-30.

Granger, Sylviane \& Paquot, Magali. (2008b). Disentangling the Phraseological Web. Dans S. Granger \& F. Meunier (dir.), Phraseology: An Interdisciplinary Perspective (p. 27-49). Amsterdam, Philadelphie : John Benjamins.

Gross, Maurice. (1981). Les bases empiriques de la notion de prédicat sémantique. Langages, 63, 7-52.

KnitTel, Marie Laurence. (2005). Some Remarks on Adjective Placement in the French NP. Probus, 17(2), 185-227.

KnitTEL, Marie Laurence. (2015a). Adjectifs et noms désadjectivaux comptables : quelles relations? Verbum («Nominalisations et Corpus»), 37(1).

KnITTEL, Marie Laurence. (2015b). Quels référents pour les noms désadjectivaux comptables? Le français moderne («La nominalisation : du fait de syntaxe aux effets de sens »), 83(1), 91-109.

Knittel, Marie Laurence \& Koenl, Aurore. (2013, septembre). L'héritage des dépendances des adjectifs par les noms désadjectivaux : ce que nous disent les données. Communication présentée au symposium international «Morphologie et Interfaces », Lille.

L'hoмme, Marie-Claude. (2008). Ressources lexicales, terminologiques et ontologiques : une analyse comparative dans le domaine de l'informatique. Revue française de linguistique appliquée, 13(1), 97-118.

Lövestam, Elin, Fuellström, Christina, Koochek, Afsaneh \& Andersson, Agneta. (2014). The Power of Language on Patient Centredness: Lin- 
guistic Devices in the Dietetic Notes of Patient Records. International Journal of Applied Linguistics, 25(2), 225-245.

Maniez, François. (2009). L'adjectif dénominal en langue de spécialité : étude du domaine de la médecine. Revue française de linguistique appliquée, 14(2), 117-130.

McNally, Louise \& Boleda, Gemma. (2004). Relational Adjectives As Properties of Kinds. Dans O. Bonami \& P. Cabredo-Hofherr (dir.), Empirical Issues in Syntax and Semantics 5. Disponible en ligne sur <www. cssp.cnrs.fr/eiss5/index_en.html> (consulté le 10 février 2015).

Née, Émilie, Sitri, Frédérique \& Veniard, Marie. (2014). Pour une approche des routines discursives dans les écrits professionnels. Dans F. Neveu, P. Blumenthal, L. Hriba, A. Gerstenberg, J. Meinschaefer \& S. Prévost (dir.), Actes du $4^{e}$ Congrès mondial de linguistique française. Disponible en ligne sur <http://dx.doi.org/10.1051/shsconf/20140801195> (consulté le 10 février 2015).

Reilly, Sheena, Bishop, Dorothy V. M. \& Tomblin, Bruce. (2014). Terminological Debate over Language Impairment in Children: Forward Movement and Sticking Points. International Journal of Language \& Communication Disorders, 49(4), 452-462.

Roche, Michel. (2006). Comment les adjectifs sont sémantiquement construits. Cahiers de Grammaire, 30, 373-87.

SoAre, Elena \& Dobrovie-Sorin, Carmen. (2002). The Romanian Supine and Adjectival Complementation. Tough Constructions. Bucharest Working Papers in Linguistics, 4(1), 75-87.

SoAre, Elena \& Giurgea, Ion. (2007, juin). When Are Adjectives Raisers? Facile to Get It. Communication présentée au «Israel Association for Theoretical Linguistics Conference», Tel-Aviv.

Tayalati, Faysal \& Van Peteghem, Marleen. (2009). Pour un traitement unitaire de l'assignation du datif en français. Lingvistica Investigationes, 32(1), 99-123.

Tutin, Agnès \& Grossman, Francis. (2002). Collocations régulières et irrégulières : esquisse de typologie du phénomène collocatif. Revue française de linguistique appliquée, 7(1), 7-25.

Vendler, Zeno. (1967). Linguistics in Philosophy. Ithaca (NY) : Cornell University Press.

Veniard, Marie. (2008). Écrire «ce qui ne va pas» dans le champ de l'enfance en danger: les mots problème(s) et difficulté(s). Carnets du Cediscor, 10, 57-77.

Walsh, Regina. (2007). A History of the Terminology of Communication Sciences and Disorders (History of Terminology: International Group on Terminology Frameworks - Communication Science and Disorders). 
Disponible en ligne sur <http://www.cplol.eu/eng/History_of_CSD.pdf> (consulté le 23 mars 2015).

Wirotius, Jean-Michel. (2012). Quel sens donner à la réadaptation? L'apport de la linguistique. Journal de réadaptation médicale («Pratique et formation en médecine physique et de réadaptation»), 32(1), 1-48. 